\title{
Avaliação dos Métodos Diagnósticos para Deficiência de GH (DGH) na Infância: IGFs, IGFBPs, Testes de Liberação, Ritmo de GH e Exames de Imagem
}

revisão

leila Pedroso de Paula

MaURo A. CZEPIELEWSKI

Serviço de Endocrinologia do

Hospital de Clínicas de Porto Alegre. Programa de PósGraduação em Ciências Médicas: Endocrinologia, Metabolismo e Nutrição da Faculdade de Medicina da Universidade Federal do Rio Grande do Sul (UFRGS), Porto Alegre, RS, Brasil.

Recebido em 11/6/2008

Aceito em 17/6/2008

\author{
RESUMO
}

O emprego das diversas metodologias diagnósticas da deficiência de hormônio de crescimento (DGH) em crianças é controverso. Neste artigo serão analisadas estas alternativas revisando a literatura e apresentando dados prospectivos obtidos pelos autores, sugerindo que a DGH seja diagnosticada empregando-se testes de triagem seguidos de testes de confirmação. Assim, recomenda-se que crianças com baixa estatura sejam avaliadas clínica e laboratorialmente para exclusão de doenças crônicas e genéticas. Naquelas com estatura $<3^{\circ}$ percentil ou velocidade de crescimento $(\mathrm{VC})<$ percentil 25, dosar IGF-1 como triagem. Se IGF-1 <-1 desvio-padrão (DP), a DGH deve ser confirmada pela ausência de resposta do hormônio de crescimento $(\mathrm{GH})$ a dois testes de estímulo (pico $<5 \mathrm{mcg} / \mathrm{L}$ ). Em paciente com fatores de risco, IGF-1 <-1 DP e um teste não-responsivo também é diagnóstico de DGH. As crianças com IGF-1 > -1 DP, devem ter a VC acompanhada e, se alterada, pode-se indicar reavaliação do eixo GH/IGF-1 excluindo ou confirmando a DGH. (Arq Bras Endocrinol Metab 2008; 52/5:734-744)

Descritores: Hormônio do crescimento; IGF-1, IGFBP-3; Teste de estímulo do GH; $\mathrm{GH}$-teste de estímulo com clonidina; Pontos de corte do $\mathrm{GH}$; Deficiência de GH

\section{ABSTRACT}

Evaluating Diagnosis Methods on Childhood GH (DGH) Deficiency: IGFs, IGFBPs, Releasing Tests, GH Rhythm and Image Exams.

The diagnostic approach to growth hormone deficiency (GHD) in children with short stature (SS) is controversial. Here we review the available methodology and present prospective data obtained in a cohort of patients with SS suggesting the use of screening test followed by the confirmation test. Thus, the children with SS should be submitted to clinical and laboratorial evaluation to exclude of chronic and genetic diseases. In addition patients with height $<3$ percentil or growth velocity < percentil 25, IGF-1 levels should be measured. If the IGF1 levels $<-1$ standard deviation (SD) compared to the age, GHD should be confirmed by two $\mathrm{GH}$-stimulations tests (peak $<5 \mathrm{mcg} / \mathrm{L}$ ). In risk factor patients, IGF-1 <-1 SD and one non-responsible GH-test, the GHD was confirmed. Children with IGF-1 > -1 SD, the growth velocity should have observed and GH/IGF-1 axis re-evaluated if the growth pattern is not satisfactory. (Arq Bras Endocrinol Metab 2008; 52/5:734-744)

Keywords: GH, IGF-1; IGFBP-3; GH-stimulation test; GH-clonidine stimulation test; ROC-curve for $\mathrm{GH}$-test; Growth hormone deficiency

\section{INTRODUÇÃo}

$\mathrm{D}$ URANTE MUITAS DÉCADAS o diagnóstico da deficiência de hormônio do crescimento (DGH) foi estabelecido em bases puramente clínicas, considerando-se, principalmente, o grau de retardo de crescimento, a velocidade de crescimento (VC), o quadro clínico característico, as deficiências hormonais associadas, a presença de doença hipotálamo-hipofisária estabelecida e a 
história familiar. A partir do isolamento do $\mathrm{GH}$ e da possibilidade de sua mensuração por radioimunoensaio, sua dosagem passou a fazer parte da rotina de avaliação de pacientes com baixa estatura (BE). Nesta situação, identificou-se rapidamente que a dosagem basal não era útil para o diagnóstico de sua deficiência, em virtude da secreção pulsátil e predominantemente noturna do hormônio de crescimento $(\mathrm{GH})$. Assim, foram sendo propostos testes de estímulo tanto fisiológicos (hipoglicemia insulínica, exercício, GHRH, ritmo de secreção de 24 horas) quanto farmacológicos (clonidina, L-dopa, arginina, propranolol, glucagon, piridostigmina e outros), sugerindo-se níveis de GH a serem atingidos (picos) em indivíduos normais em cada um deles. Com o surgimento de metodologias mais sensíveis e específicas, foram se tornando possíveis dosagens cada vez menores de GH e de suas respectivas formas moleculares. Estes avanços levaram à proposição, como normais, de picos cada vez menores de GH e a necessidade também de estabelecimento de padrões de referência a partir de moléculas sintéticas do hormônio, com peso e estrutura molecular bem definidos, especialmente a forma com $22 \mathrm{kDa}$.

Com a demonstração de que uma parte importante das ações do GH se faz a partir da produção endócrina, parácrina e autócrina de fatores de crescimento (insulin-like growth factor-1 and 2 [IGF-1 e IGF-2]) e da importância de suas proteínas ligadoras circulantes (insulin-like growth factor binding protein, IGFBPs), a mensuração destas também passou a ser proposta como ferramenta diagnóstica da DGH.

\section{Exames de rastreamento, triagem ou screening}

Por definição, o teste diagnóstico a ser utilizado no rastreamento da DGH deve ser sensível, ou seja, deve oferecer a possibilidade de incluir todos os verdadeiros portadores do distúrbio em uma população avaliada. Além da alta sensibilidade, deve ser de fácil aplicação, baixo custo e pouco invasivo. Usualmente, os testes de triagem são indicados para doenças graves que apresentam fase pré-clínica e cujo diagnóstico, nesta fase, oferece relação custo-benefício favorável. O diagnóstico da DGH em pacientes com BE preenche, sem dúvida, estes critérios para utilização de testes de triagem.

\section{Exames confirmatórios}

Depois de serem realizados testes de rastreamento, faz-se necessário utilizar testes que confirmem com segurança o diagnóstico da DGH. Nesta situação, é preciso empregar testes diagnósticos específicos. Quanto maior a especifici- dade de um teste, maior é a chance de que pessoas sem a doença sejam excluídas pelo teste. Estes aspectos são muito importantes, uma vez que a DGH envolve tratamento por tempo prolongado, com custo elevado e necessita de controles médicos periódicos. Assim, se em um momento de triagem faz-se necessários os testes sensíveis, para a confirmação diagnóstica da DGH, impõe-se o emprego de testes e pontos de corte específicos.

Conforme apresentado na Tabela 1 , são úteis para o diagnóstico da DGH: exames de imagem, como ressonância magnética (RM) e tomografia computadorizada, e exames laboratoriais, como IGF-1 e IGF-2, as suas proteínas carreadoras dos tipos 2 e 3 (insulin-like growth factor binding proteins 2 and 3 [IGFBP-2 e IGFBP-3]), assim como a subunidade ácido-lábil (ALS), a concentração integrada de GH em 12 ou 24 horas (CI-GH) e os testes de estímulo da secreção de GH

Neste trabalho foram revisadas as principais estratégias diagnósticas para DGH na infância, salientando as evidências quanto à utilidade destes métodos e como interpretá-los e, a partir da experiência do grupo, suge-

Tabela 1. Métodos de triagem e confirmação no diagnóstico da DGH em crianças.

\begin{tabular}{l} 
Triagem ou screening \\
Características clínicas \\
Ao nascimento: defeitos de linha média, micropênis, \\
criptorquidia, hipoglicemia, icterícia prolongada \\
BE grave, abaixo de 3 DP da média ou 1,5 DP abaixo da \\
altura-alvo \\
Sem BE atual ou inicial, porém com VC abaixo do \\
percentil 25 para a idade \\
Região frontal proeminente, baixa implantação do nariz, \\
deposição de gordura abdominal, voz fina \\
Diagnóstico de outra doença hipotálamo-hipofisária \\
IGF-1 e IGFBP-3 \\
Valores menores que 1 ou 2 DP para a média populacio- \\
nal \\
Textes de estímulo do GH \\
Estímulo: arginina, clonidina, glucagon, insulina e L-dopa \\
Ensaios monoclonais ou mono-policlonais, por quimiolumi- \\
nescência, imunofluorimétricos ou imunorradiométricos \\
Resposta normal: maior que 5 mcg/L \\
Concentração integrada de GH em 12 ou 24 horas \\
Útil em centros de referência e pesquisa \\
Valores normais médios maiores que 3,3 mcg/L \\
Imagem da região hipotálamo-hipofisária \\
RM de alta resolução com imagens em T1 e T2, incluindo \\
reconstrução sagital e coronal \\
\hline
\end{tabular}


riu-se abordagem diagnóstica prática e útil para a rotina assistencial de pacientes com BE.

\section{EXAMES DE RASTREAMENTO}

\section{Fatores de crescimento e proteínas carreadoras}

\section{IGFs}

São descritos três ligantes aos receptores tipo IGF, quais sejam, IGF-1, IGF-2 e insulina. Todos interferem no crescimento. A insulina e o IGF-2 são importantes predominantemente no período fetal, enquanto o IGF-1 tem ação tanto intra-uterina quanto pós-natal (1). Os níveis de IGF-1 e IGF-2 são GH-dependentes; o IGF-1 mais do que o IGF-2, e ambos circulam em níveis relativamente constantes durante o dia. Assim sua dosagem prescinde de múltiplas coletas e da necessidade de testes de estímulo (2-4).

\section{IGF-1}

Quando se avalia o valor basal de IGF-1 de uma criança é importante que se conheçam as potenciais limitações e/ou fatores que possam interferir nos métodos de sua mensuração. A seguir serão apresentadas algumas destas observações, a partir de dados de literatura.

As IGFBPs podem interferir nas dosagens de IGF-1 por radioimunoensaio ou bioensaio, especialmente em pacientes urêmicos, naqueles em que a relação IGFBP/ IGF-l é relativamente alta e em pacientes que apresentam valores nos extremos do ensaio, como os com DGH grave. Esta interferência pode ser contornada separando o IGF-1 das IGFBPs por cromatografia ácido-gel, utilizando o método ELISA ou o IRMA tipo "sanduíche" $(2,3,5,6)$.

A concentração de IGF- 1 varia com a idade, aumentando gradualmente durante a infância, atingindo seu pico, em média, aos 13 anos nos meninos, e de 11 a 13 anos nas meninas, ou no estágio IV da puberdade. Até os 5 anos de idade os valores normais são muito baixos, dificultando a discriminação. Por isso, recomenda-se analisar o resultado não pelo valor absoluto, mas sim pelo DP da média, conforme o sexo e a idade $(2,3,5,7)$.

Os valores de referência para o IGF-1 são definidos por idade e sexo e, como sugerido por Saenger e cols., as crianças com desordens do crescimento seguidamente têm retardo no desenvolvimento físico e/ou puberal, sendo mais adequado comparar os resultados do IGF-1 utilizando o padrão para a idade óssea $(3,8)$.
Pode ocorrer variabilidade por diferentes ensaios e diferentes populações, sugerindo-se a utilização de padrão para a calibração da Organização Mundial de Saúde (OMS) - IRR 87/518 - e a criação de valores de referência específicos para cada população e/ou laboratório $(3,7)$.

Os níveis de IGF-1 são influenciados pela presença de doenças crônicas ou desnutrição e também sofrem ação dos hormônios tireoidianos, gonadais e adrenais $(1,5)$.

A deficiência de IGF-1 não evidencia o nível do defeito do eixo GH/IGF-1, podendo sua redução estar associada à deficiência de produção e/ou secreção, alteração na bioatividade ou resistência à ação do $\mathrm{GH}(2,5,7)$.

Em crianças que receberam radioterapia sobre o crânio e naquelas que apresentam tumores cerebrais, os níveis de IGF-1 demoram a diminuir, mesmo com redução da secreção de $\mathrm{GH}(5,9,10)$.

Considerando estes aspectos, o Consensus Guideline da GH Research Society, de 2000, propõe a utilização do ponto de corte do IGF-l inferior a 2 DP abaixo da média para a idade ( $-2 \mathrm{DP})$ como sugestivo de anormalidade do eixo GH (7). Em um trabalho recente do grupo, foi redefinido este ponto de corte, quando utilizou-se a medida basal de IGF-1 como teste de screening para a suspeita da DGH. Em uma coorte de 851 crianças com baixa estatura, 270 participaram de estudo de teste diagnóstico aninhado. Utilizou-se o método de curva ROC (receiver operating characteristics) para determinar pontos de corte da dosagem basal de IGF-1 (imunorradiométrico com extração, DSL-5600) e do pico de resposta do GH (quimioluminescência, Immulite - DPC) em resposta ao menos a um teste de estímulo de GH, seja com clonidina (GH-Clo) ou com insulina. Foram diagnosticados 51 pacientes com DGH. A melhor acurácia observada foi com o ponto de corte de -1 DP do IGF-1, em crianças sem doença crônica, síndrome genética ou má nutrição $(92,9 \%$ sensibilidade e $84,2 \%$ especificidade) e está representada na Tabela 2 (11). Nesta mesma tabela pode-se analisar a sensibilidade e a especificidade dos pontos de corte da dosagem basal de IGF-1, IGF-2, IGFBP-2 e IGFBP-3 em diferentes estudos, utilizando-se populações e padrõesouro distintos, conforme dados da literatura (2,11-13).

Juul e cols., avaliaram 108 pacientes previamente diagnosticados como deficientes no momento do reteste e compararam a utilidade da medida do IGF-1 na reavaliação destes pacientes. O único grupo com deficiência de três ou mais hormônios hipofisários, além do $\mathrm{GH}$, foi aquele em que todos os pacientes apresentaram valores 
Tabela 2. Performance das IGFs e IGFBPs no diagnóstico da DGH, expresso pela sensibilidade e especificidade, pontos de corte e padrão-ouro utilizado.

\begin{tabular}{|c|c|c|c|c|c|c|c|}
\hline $\begin{array}{l}\text { Estudo/ano } \\
\text { (referência) }\end{array}$ & Pacientes & $\begin{array}{l}\text { Ponto de } \\
\text { corte GH } \\
(\mathrm{mcg} / \mathrm{L})\end{array}$ & $\begin{array}{c}\text { Tipo de ensaio } \\
\text { GH }\end{array}$ & Padrão-ouro & $\begin{array}{l}\text { Ponto de } \\
\text { corte IGF-1 } \\
\text { (DP) }\end{array}$ & Sensibilidade & Especificidade \\
\hline \multirow{3}{*}{$\begin{array}{l}\text { de Paula e } \\
\text { cols., } 2007 \\
\text { (11) }\end{array}$} & \multirow{3}{*}{$\begin{array}{c}270 \text { crianças } \\
\text { com BE (51 DGH } \\
\text { e } 219 \text { com BEVN) }\end{array}$} & \multirow[t]{3}{*}{10} & \multirow{3}{*}{$\begin{array}{l}\text { Monopoliclonal } \\
\text { quimiolumi- } \\
\text { nescência }\end{array}$} & \multirow{3}{*}{$\begin{array}{l}\text { BE e baixa VC, } \\
\text { GH-Clo sem e } \\
\text { com priming e } \\
\text { GH-insulina }\end{array}$} & $-2 \mathrm{DP}$ & $14,3 \%$ & $98,9 \%$ \\
\hline & & & & & $-1,5 \mathrm{DP}$ & $64,3 \%$ & $93,7 \%$ \\
\hline & & & & & $-1 \mathrm{DP}$ & $92,9 \%$ & $84,2 \%$ \\
\hline \multirow[t]{6}{*}{$\begin{array}{l}\text { Nunez e } \\
\text { cols., } 1996 \\
\text { (12) }\end{array}$} & \multirow[t]{6}{*}{$\begin{array}{c}104 \text { crianças } \\
\text { com BE, } \\
\text { sensibilidade de } \\
\text { DGH completa e } \\
\text { parcial }\end{array}$} & \multirow[t]{6}{*}{$7-10$} & \multirow[t]{6}{*}{ Policlonal RIE } & \multirow[t]{4}{*}{$\begin{array}{l}\text { GH-arginina e } \\
\text { insulina e } \\
\text { arginina e } \\
\text { L-dopa com } \\
\text { priming }\end{array}$} & $-2 \mathrm{DP}$ & $50 / 21 \%$ & $76 \%$ \\
\hline & & & & & $-1 \mathrm{DP}$ & $88 / 71 \%$ & $54 \%$ \\
\hline & & & & & $\begin{array}{c}-2 \text { DP } \\
\text { IGFBP-3 }\end{array}$ & $31 / 29 \%$ & $82 \%$ \\
\hline & & & & & $\begin{array}{c}-1 \text { DP } \\
\text { IGFBP-3 }\end{array}$ & $88 / 57 \%$ & $43 \%$ \\
\hline & & & & \multirow[t]{2}{*}{ Cl-GH 12 horas } & $\begin{array}{c}-2 \text { DP IGF-1 } \\
\text { e IGFBP-3 }\end{array}$ & $25 / 0 \%$ & $97 \%$ \\
\hline & & & & & $\begin{array}{c}-1 \text { DP IGF-1 } \\
\text { e IGFBP-3 }\end{array}$ & $81 / 50 \%$ & $74 \%$ \\
\hline \multirow{5}{*}{$\begin{array}{l}\text { Juul e cols., } \\
1997 \text { (13) }\end{array}$} & \multirow{5}{*}{$\begin{array}{l}108 \text { pacientes } \\
\text { retestados com } \\
\text { diagnóstico } \\
\text { prévio de DGH } \\
1.430 \text { crianças e } \\
\text { adultos } \\
\text { saudáveis }\end{array}$} & \multirow[t]{5}{*}{7,5} & \multirow[t]{5}{*}{ Policlonal RIE } & \multirow[t]{5}{*}{ GH-Clo } & $-2 \mathrm{DP}$ & $75,9 \%$ & $72 \%$ \\
\hline & & & & & $-1 \mathrm{DP}$ & $90 \%$ & $50 \%$ \\
\hline & & & & & IGFBP-3 & $68,4 \%$ & $79 \%$ \\
\hline & & & & & $-2 \mathrm{DP}$ & & \\
\hline & & & & & $\begin{array}{l}\text { IGFBP-3 } \\
-1 \mathrm{DP}\end{array}$ & $85 \%$ & $60 \%$ \\
\hline \multirow[t]{2}{*}{$\begin{array}{l}\text { Bussières e } \\
\text { cols., } 2000 \\
\text { (2) }\end{array}$} & $\begin{array}{l}\text { Só crianças: } \\
\text { - } 68 \text { com BEl } \\
\text { - } 28 \text { com DGH }\end{array}$ & 10 & \multirow[t]{2}{*}{$\begin{array}{l}\text { Imunorradio- } \\
\text { métrico }\end{array}$} & $\begin{array}{c}\text { DGH - dois } \\
\text { testes alterados }\end{array}$ & $\begin{array}{c}-2 \text { DP em } \\
\text { relação aos } \\
\text { normais }\end{array}$ & $84 \%$ & $57 \%$ \\
\hline & $\begin{array}{l}\text { familiar ou por } \\
\text { defeito de haste } \\
\text { - } 15 \text { DGH e } \\
\text { craniofaringioma } \\
\text { - } 168 \text { normais }\end{array}$ & 10 & & BEI & $\begin{array}{c}-2 \mathrm{DP} \text { em } \\
\text { relação aos } \\
\text { com BEl }\end{array}$ & $72 \%$ & $95 \%$ \\
\hline
\end{tabular}

$\mathrm{BE}$ = baixa estatura; $\mathrm{DGH}=$ deficientes de hormônio do crescimento; $\mathrm{BEVN}=$ baixa estatura variante da normalidade; $\mathrm{VC}=$ velocidade de crescimento; $\mathrm{GH}-\mathrm{Clo}=$ teste de estímulo do GH pela clonidina; $\mathrm{DP}=$ desvio-padrão; $\mathrm{Cl}-\mathrm{GH}$ = concentração integrada de $\mathrm{GH}$ em 24 horas; $\mathrm{BEl}=$ baixa estatura idiopática.

de IGF-1 abaixo de -2 DP (13). Assim, valores de IGF-1 abaixo de -2 DP são característicos de pacientes com graves deficiências hormonais, sendo discutível sua utilidade como teste de triagem. Pelo contrário, pequena redução de IGF-1 (-1 DP), em pacientes nos quais foram excluídas doenças sistêmicas, genéticas e distúrbios nutricionais, pode ser útil na triagem da DGH, uma vez que todos os pacientes portadores desta deficiência seriam diagnosticados por testes confirmatórios. (11)

\section{IGF-1 livre}

A dosagem do IGF-1 livre também é mais alta na puberdade, entretanto, a relação IGF-1 livre/IGF-1 total é maior no primeiro ano de vida e isso quiçá possa contribuir para a VC acelerada desta fase $(14,15)$. Talvez a medida de IGF-1 livre só apresente vantagem sobre a medida do IGF-l total no primeiro ano de vida (4). 


\section{IGF-2}

Os níveis de IGF-2 são menos dependentes da idade e não variam muito após o primeiro ano de vida extrauterina. Todavia, um estudo de Rosenfeld e cols. demonstrou níveis de IGF-2 normais em $48 \%$ de 68 crianças deficientes de GH (15). Neste mesmo estudo apenas $4 \%$ dos deficientes apresentavam níveis normais de IGF-1 e IGF-2, concomitantemente, enquanto apenas $0,5 \%$ das 197 crianças sem BE apresentava ambos os níveis (IGF-l e IGF-2) diminuídos. Aparentemente, a dosagem do IGF-2 é mais útil do segundo ao quinto ano de vida, em associação com a dosagem do IGF-1, embora seja pouco utilizado na prática clínica (16).

\section{IGFBPs}

As IGFBPs (principalmente o complexo ternário IGF-1/ IGFBP-3/subunidade ácido-lábil-ALS) aumentam a meia-vida das IGFs, inibindo sua proteólise e depuração, contribuindo, assim, com o armazenamento de IGFs (depósito biológico). Este fato fica evidente quando avalia-se a meia-vida: de 10 a 20 minutos do IGF-1 livre comparado com 15 horas do complexo ternário. Os principais fatores implicados na regulação das IGFs são: nível de GH/IGF-1, idade, estágio puberal, jejum e desnutrição, reduzindo a concentração de IGFBP-3 e aumentando as de IGFBP-1 e IGFBP-2, reduzindo assim a longo prazo a meia-vida das IGFs, porém aumentando o transporte por meio do endotélio capilar e, conseqüentemente, aumentando o aporte de IGFs livres nos tecidos-alvo $(2,4)$.

\section{IGFBP-2}

O IGFBP-2 está aumentado na maioria dos pacientes com DGH, porém não tem poder discriminatório melhor do que a dosagem basal de IGF-1 (16). Aparentemente a relação IGFBP-2/IGF-1 tem maior capacidade de diferenciar os pacientes com deficiência dos sem DGH, especialmente se esta relação se encontra 2 DP acima da média (5).

\section{IGFBP-3}

A maioria dos estudos que avaliam a utilidade da dosagem da IGFBP-3 no diagnóstico da DGH não foi capaz de provar vantagem em relação ao IGF-l. Todavia, a dosagem combinada de ambos parece melhorar a eficácia diagnóstica, principalmente nos primeiros anos de vida, quando os valores normais baixos de IGF-1 dificultam a discriminação (17).

\section{ALS}

A subunidade ácido-lábil da IGFBP-3 também é estável durante o dia, varia conforme a idade e a puberdade. Estudo de Juul e cols. demonstrou que a dosagem da ALS apresenta sensibilidade de $72 \%$ no diagnóstico da $\operatorname{DGH}(6)$.

\section{EXAMES CONFIRMATÓRIOS}

\section{Concentração integrada de GH em 24 horas}

Para a avaliação da secreção espontânea de $\mathrm{GH}$, inicialmente, foram utilizados diversos critérios de quantificação: a concentração média do GH no período, o pico máximo, a área sob a curva, o número de picos acima de $5 \mathrm{mcg} / \mathrm{L}$ e a área dos picos acima de 5 $\mathrm{mcg} / \mathrm{L}$ (18). Considera-se como normal a secreção de GH com níveis acima de $3,3 \mathrm{mcg} / \mathrm{L}$. Porém, mesmo crianças com estatura e VC normais podem apresentar valores abaixo deste ponto de corte. No estudo de Zadik e cols., 2,5\% de 119 crianças normais de 7 a 19 anos apresentaram níveis de CI-GH abaixo de 3,2 $\mathrm{mcg} / \mathrm{L}$. Os pacientes púberes (Tanner II-V) apresentam CI-GH significativamente maior do que os prépuberais $(5,8 \times 4,4 \mathrm{mcg} / \mathrm{L})$. A reprodutibilidade da CI-GH é significativamente melhor do que a dos testes de estímulo (19). Aparentemente, a diferença na secreção de $\mathrm{GH}$ entre os deficientes e as crianças normais ou as crianças com baixa estatura idiopática (BEI) se acentua durante a noite $(1,1 \times 3,9 \times 4,4 \mathrm{mcg} / \mathrm{L})$ e a concentração integrada de $\mathrm{GH}$ durante a noite pode ser melhor teste diagnóstico do que a CI-GH em 24 horas. A CI-GH também pode diagnosticar a controversa disfunção neuro-secretória (9).

\section{Teste de estímulo do GH}

Uma série de estímulos fisiológicos e farmacológicos tem sido utilizada como testes para demonstrar a suficiência da secreção do GH. A maioria dos países estabeleceu critérios para o diagnóstico de DGH fundamentados em respostas máximas do $\mathrm{GH}$ (picos) "normais" arbitrários. O pico maior do que $10 \mathrm{mcg} / \mathrm{L}$ é usualmente aceito como resposta normal aos estímulos (7). Entretanto, quando se testou crianças sem BE, verificou-se que poderiam apresentar respostas inferiores a $10 \mathrm{mcg} / \mathrm{L}$ (20). Vários estudos têm demonstrado que análogos de $\mathrm{GH}$ diferentes da forma de $22 \mathrm{kDa}$ e fragmentos podem interagir como agonistas fracos ou antagonistas do receptor de GH (1). Apesar de o uso de 
novos ensaios, nos quais os valores de $\mathrm{GH}$ medido sejam mais baixos do que os dos ensaios anteriores, por medirem predominantemente o $\mathrm{GH}$ biologicamente ativo $(22 \mathrm{kDa})$ na circulação, não se modificou este ponto de corte. Além disso, pode ocorrer variação dos níveis de GH, dependendo também do tipo de ensaio empregado, da preparação de referência, da pureza do traçador de $\mathrm{GH}$, da especificidade do anticorpo antiGH (monoclonal versus policlonal) e do método básico de medida (21). É importante que o médico endocrinologista, ao interpretar o teste de estímulo de GH, esteja ciente sobre o tipo de ensaio utilizado, a especificidade do anticorpo e a preparação de referência.

\section{Tipo de estímulo}

Não há consenso a respeito da utilização de determinado teste como padrão-ouro, embora se considere que a associação GHRH + arginina seja o estímulo mais potente (5). Este teste é pouco disponível em nosso meio. Como pode apresentar resposta normal quando a causa de DGH for hipotalâmica, não deve ser usado em pacientes nos primeiros anos após irradiação craniana, pelo elevado número de falsos-negativos (10).

Os testes atualmente recomendados incluem a arginina, a clonidina, o glucagon, a insulina e a L-dopa. Em crianças menores de 2 anos têm-se preferido o teste do glucagon, pelo perfil de possíveis efeitos colaterais (22). Não se recomenda o uso do GH estimulado pelo exercício, apesar de ainda muito popular em nosso meio. $\mathrm{O}$ estudo de Ghigo e cols., com 472 crianças com VC normal, utilizou um teste com exercício físico rigoroso, com bicicleta ergométrica com trabalho e carga progressivos, de acordo com o protocolo de James, coletas de sangue para dosagem de GH de 15 em 15 minutos, por 2 horas, e observou que $36,4 \%$ destas crianças normais não respondiam ao teste (23).

\section{Método básico de medida}

Quando foram comparados os valores de GH da mesma amostra, dosados por diferentes ensaios, observouse que os ensaios imunorradiométricos produziam resultados de cerca de $60 \%$ dos por radioimunoensaio, talvez por maior especificidade do primeiro pela forma com $22 \mathrm{kDa}(24)$.

\section{Preparação de referência}

Recomenda-se o uso do GH recombinante humano de $22 \mathrm{kDa}(88 / 624$, potência $3 \mathrm{UI}=1 \mathrm{mg})$.

\section{Especificidade do anticorpo anti-GH}

Os ensaios que utilizam anticorpos monoclonais dificilmente dosam as isoformas de $\mathrm{GH}$ e por este motivo produzem valores muito mais baixos do que os com anticorpos policlonais $(7,21)$.

\section{Pico de GH nos testes de estímulo (ponto de corte)}

A análise do banco de dados do KIGS (Kabi International Growth Study) de 16.327 crianças com DGH demonstrou crescimento igual no grupo de pacientes com resposta aos testes maior do que $10 \mathrm{mcg} / \mathrm{L}$ ao dos com resposta entre 5 e $10 \mathrm{mcg} / \mathrm{L}$. A melhor VC e o ganho de altura durante todo o tratamento só foi maior nos grupos com resposta ao teste até $5 \mathrm{mcg} / \mathrm{L}$ e nos pacientes com pan-hipopituitarismo (25). Estudos que retestam crianças com diagnóstico de DGH isolada após o final do crescimento têm visto que cerca de $50 \%$ não têm o resultado dos primeiros testes confirmado no reteste. Isso parece refletir a utilização de critérios diagnósticos inapropriados, entre os quais o do ponto de corte do pico de $\mathrm{GH}$ ao teste de estímulo (26). Os dados do presente estudo, associados à revisão da literatura, confirmam a necessidade da modificação do ponto de corte da resposta do GH para valores em torno de 4 a $5 \mathrm{mcg} / \mathrm{L}$, quando este for dosado utilizando o ensaio quimioluminescente monopoliclonal (Imulite, DPC), como pode-se observar na Tabela 3. Além disso, eles demonstram que este valor, previamente validado em resposta à clonidina, coincide com o valor, quando o estímulo utilizado é a hipoglicemia insulínica (11).

\section{Priming}

Ao contrário de estudos anteriores, em que o valor do ponto de corte da resposta do GH se modificava quando era antecedido por esteróides sexuais (20), no nosso trabalho observa-se amplificação significativa da resposta do GH apenas no grupo de pacientes com baixa estatura variante da normalidade e não nos deficientes. Nesta casuística, os testes de estímulo do GH foram precedidos de priming que consistiu da administração de testosterona $50 \mathrm{mg}$ intramuscular 7 e 2 dias antes do teste nos meninos, e estrógenos conjugados $1,2 \mathrm{mcg} /$ $\mathrm{m}^{2} /$ dia por 3 dias antes do teste nas meninas, quando tinham 7 anos ou mais e estágio puberal I ou II. Este fato fez que o ponto de corte nos testes sem e nos com priming não fosse muito diferente, e demonstrou que 
Tabela 3. Avaliação do ponto de corte da resposta do GH aos testes de estímulo no diagnóstico da DGH, expresso pela sensibilidade e pela especificidade.

\begin{tabular}{|c|c|c|c|c|c|c|c|}
\hline $\begin{array}{l}\text { Autor/ano } \\
\text { (referência) }\end{array}$ & Pacientes & $\begin{array}{l}\text { Tipo de } \\
\text { ensaio GH }\end{array}$ & Padrão-ouro & Tipo de teste & $\begin{array}{l}\text { Ponto de } \\
\text { corte do GH } \\
\text { (mcg/L) }\end{array}$ & Sensibilidade & Especificidade \\
\hline \multirow{9}{*}{$\begin{array}{l}\text { De Paula e } \\
\text { cols., } 2007 \\
\text { (11) }\end{array}$} & \multirow{9}{*}{$\begin{array}{c}270 \\
\text { crianças } \\
\text { com BE (51 } \\
\text { DGH e } 219 \\
\text { com BEVN) }\end{array}$} & \multirow{9}{*}{$\begin{array}{l}\text { Monopoli- } \\
\text { clonal } \\
\text { quimiolumi- } \\
\text { nescência }\end{array}$} & \multirow{9}{*}{$\begin{array}{l}\text { BE e baixa } \\
\text { VCGH clonidina } \\
\text { sem e com } \\
\text { priming e } \mathrm{GH} \\
\text { insulina }\end{array}$} & \multirow{3}{*}{$\begin{array}{c}\mathrm{GH}-\mathrm{Clo} \\
\text { sem priming }\end{array}$} & 10 & $100 \%$ & $53,9 \%$ \\
\hline & & & & & 7 & $100 \%$ & $73 \%$ \\
\hline & & & & & 4 & $95 \%$ & $93,4 \%$ \\
\hline & & & & \multirow{3}{*}{$\begin{array}{l}\text { GH-Clo com } \\
\text { priming }\end{array}$} & 10 & $100 \%$ & $75 \%$ \\
\hline & & & & & 7 & $96,3 \%$ & $93,3 \%$ \\
\hline & & & & & 5 & $96,3 \%$ & $100 \%$ \\
\hline & & & & \multirow{3}{*}{$\begin{array}{l}\text { GH total } \\
\text { (insulina e } \\
\text { clonidina) }\end{array}$} & 10 & $100 \%$ & $76,5 \%$ \\
\hline & & & & & 7 & $97,9 \%$ & $92,4 \%$ \\
\hline & & & & & 5 & $97,9 \%$ & $97,6 \%$ \\
\hline \multirow{4}{*}{$\begin{array}{l}\text { Silva e cols., } \\
2002 \text { (27) }\end{array}$} & \multirow{4}{*}{$\begin{array}{c}30 \\
\text { crianças } \\
\text { normais e } \\
26 \text { DGH } \\
1-22 \text { anos }\end{array}$} & \multirow{4}{*}{$\begin{array}{l}\text { Monoclonal } \\
\text { imunofluorimé- } \\
\text { trico }\end{array}$} & \multirow{4}{*}{$\begin{array}{l}14 \text { com pan- } \\
\text { hipopituitarismo, } \\
8 \text { com defeitos } \\
\text { moleculares e } \\
\text { demais com } \\
\text { interrupção de } \\
\text { haste }\end{array}$} & \multirow{4}{*}{$\begin{array}{l}\mathrm{GH}-\mathrm{Clo} \text { e } \mathrm{GH} \\
\text { insulina }\end{array}$} & 10 & $100 \%$ & $66,7 \%$ \\
\hline & & & & & 7 & $100 \%$ & $83,3 \%$ \\
\hline & & & & & 5,5 & $100 \%$ & $90 \%$ \\
\hline & & & & & 3,3 & $100 \%$ & $93,3 \%$ \\
\hline \multirow[t]{2}{*}{$\begin{array}{l}\text { Martínez e } \\
\text { cols., } 2000 \\
\text { (28) }\end{array}$} & \multirow{2}{*}{$\begin{array}{l}15 \text { DGH } \\
\text { pré-pu- } \\
\text { berais e } 44 \\
\text { com BEl }\end{array}$} & & & $\begin{array}{l}\text { GH arg-clo + } \\
\text { placebo de } \\
\text { priming }\end{array}$ & 3,7 & $73 \%$ & $95 \%$ \\
\hline & & & & $\begin{array}{l}\text { GH arg-clo } \\
\text { com priming }\end{array}$ & 8,3 & $87 \%$ & $98 \%$ \\
\hline \multirow{2}{*}{$\begin{array}{l}\text { Chaler e } \\
\text { cols., } 2006 \\
(29)\end{array}$} & \multirow{2}{*}{$\begin{array}{c}39 \\
\text { pacientes } \\
\text { DGH e } 11 \\
\text { com BEVN }\end{array}$} & & \multirow{2}{*}{$\begin{array}{c}\text { Escore do DP da } \\
\text { VC no } 1^{\circ} \text { ano } \\
\text { de tratamento } \\
\text { com GH }\end{array}$} & \multirow[t]{2}{*}{$\begin{array}{l}\mathrm{GH}-\mathrm{Clo} \text { e } \\
\text { arginina }\end{array}$} & 10,8 & \multirow{2}{*}{\multicolumn{2}{|c|}{$\begin{array}{c}\text { Não descreve a sensibilidade e } \\
\text { especificidade. } \\
\text { Apenas relata o ponto de corte } \\
\text { de maior acurácia para cada } \\
\text { tipo de ensaio, nos mesmos } \\
\text { pacientes. }\end{array}$}} \\
\hline & & Monoclonal & & & 5,4 & & \\
\hline
\end{tabular}

$\mathrm{BE}$ = baixa estatura; $\mathrm{DGH}=$ deficientes de hormônio do crescimento; $\mathrm{BEVN}=$ baixa estatura variante da normalidade; $\mathrm{GH}-\mathrm{Clo}=$ teste de estímulo com clonidina; $\mathrm{BEI}=$ baixa estatura idiopática; GH arg-clo = teste de estímulo com arginina + clonidina; DP = desvio-padrão; VC = velocidade de crescimento.

quando este ponto de corte é reduzido para $5 \mathrm{mcg} / \mathrm{L}$ se reduz a necessidade do priming, a fim de amplificar a resposta e acentuar esta diferenciação. (11)

A tabela 4 resume os prós e os contras dos métodos diagnósticos utilizados na avaliação do eixo $\mathrm{GH} /$ IGF-1.

\section{Diagnóstico por imagem da região hipotálamo-hipofisária}

O diagnóstico por imagem da região hipotálamo-hipofisária está sempre indicado no momento em que se demonstra a DGH, seja isolada ou associada a outras deficiências hormonais hipofisárias. Este diagnóstico poderá demonstrar alterações que exijam manejo espe- cífico, como lesões tumorais, inflamatórias ou congênitas, entre as quais se destacam o craniofaringioma e a hidrocefalia congênita. Com a descoberta de diversos genes relacionados à morfogênese hipofisária, a identificação de suas mutações vem sendo associada a diversas alterações da região hipotálamo-hipofisária e outras regiões do sistema nervoso central (SNC). A caracterização anatômica destas alterações também é indicação de investigação por imagem. (31)

Com estes objetivos deve-se preferir a RM de alta resolução, com cortes de até $3 \mathrm{~mm}$ de espessura, nos planos coronal e sagital, com o uso de gadolíneo e, se necessário, com a criança sedada, obtendo-se seqüências em Tl e T2. Na seqüência em T1, deve-se realizar 
Tabela 4. Prós e contras dos métodos diagnósticos utilizados na avaliação do eixo GH/IGF-1.

\begin{tabular}{|c|c|c|}
\hline Método diagnóstico & Prós & Contras \\
\hline GH basal & Quando elevado pode sugerir resistência. & $\begin{array}{l}\text { Não reflete a suficiência de GH - secreção } \\
\text { pulsátil. }\end{array}$ \\
\hline IGF-1 e IGFBP-3 & $\begin{array}{l}\text { Niveis mais estáveis que GH. } \\
\text { Maior reprodutibilidade. } \\
\text { Tabelas padronizadas de níveis normais para sexo } \\
\text { e idade. }\end{array}$ & $\begin{array}{l}\text { Variam conforme: idade, idade óssea, estágio } \\
\text { puberal, sexo, estado nutricional e doenças } \\
\text { orgânicas crônicas. } \\
\text { Não evidencia "o nível" do defeito do eixo. }\end{array}$ \\
\hline $\begin{array}{l}\text { Concentração integra- } \\
\text { da de GH }\end{array}$ & $\begin{array}{l}\text { Sensibilidade e especificidade semelhantes aos } \\
\text { testes de GH. } \\
\text { Pesquisas científicas. } \\
\text { Maior reprodutibilidade. }\end{array}$ & $\begin{array}{l}\text { Necessita hospitalização. } \\
\text { Custo elevado. } \\
\text { Inconveniente provocado ao paciente. } \\
\text { Discrepância entre os testes de estímulo de } \\
\text { GH e o IGF-1. }\end{array}$ \\
\hline $\begin{array}{l}\text { Testes de estímulo da } \\
\text { secreção de GH }\end{array}$ & $\begin{array}{l}\text { Confirmam a DGH quando o diagnóstico é } \\
\text { suspeito em bases auxológicas ou em um fator } \\
\text { predisponente. } \\
\text { Menor dependência de fatores nutricionais ou } \\
\text { doenças crônicas do que IGF-1. }\end{array}$ & $\begin{array}{l}\text { Diferentes protocolos e estímulos farmacológi- } \\
\text { cos. } \\
\text { Baixa reprodutibilidade. } \\
\text { Depende de: idade, peso corporal, estágio } \\
\text { puberal. } \\
\text { Picos de GH "normais" arbitrários. } \\
\text { Variação do GH depende: tipo de ensaio } \\
\text { empregado, preparação de referência, } \\
\text { pureza do traçador de GH, anticorpo mono X } \\
\text { policlonal, método básico de medida. } \\
\text { Sem consenso para priming. } \\
\text { Respostas normais ao reteste. }\end{array}$ \\
\hline $\begin{array}{l}\text { Avaliação genética } \\
\text { (mutação/del. genes do } \\
\text { GHRH, GH, PROP-I/PIT I/ } \\
\text { HESX1/LHX 3/LHX 4/SOX) }\end{array}$ & Técnica promissora. & $\begin{array}{l}\text { Custo elevado. } \\
\text { Difícil acessibilidade. }\end{array}$ \\
\hline $\begin{array}{l}\text { GH urinário } \\
\text { IGF-II } \\
\text { IGFBP-2 } \\
\text { ALS }\end{array}$ & $\begin{array}{l}\text { Podem ser úteis quando utilizados em combina- } \\
\text { ção com outros testes. }\end{array}$ & Não são diagnósticos. \\
\hline
\end{tabular}

sempre a reconstrução sagital, procurando identificar quiasma óptico, sulcos e bulbos olfatórios, infundíbulo, tecido adenohipofisário e neuro-hipófise, que se apresenta hiperintensa. A seqüência em T2, no plano coronal, é particularmente útil para a caracterização da sela vazia e das lesões císticas hipofisárias. A tomografia computadorizada de sela túrcica ainda apresenta uma única vantagem em relação à $\mathrm{RM}$, que é a possibilidade de mais bem avaliar estruturas ósseas e calcificadas, o que é particularmente importante no diagnóstico de craniofaringeoma.

Os pacientes com DGH podem apresentar os seguintes achados na RM: hipófise normal, hipófise hipoplásica ou pequena ( $<3 \mathrm{~mm}$ de altura), sela vazia, hipoplasia hipofisária associada à neuro-hipófise ectópica e agenesia parcial ou completa da haste hipofisária. Recentemente, revisão de 13 estudos sobre a RM na avaliação de pacientes com DGH demonstrou prevalência variável de $50 \%$ a $100 \%$ de neuro-hipófise ectópica nos pacientes com deficiências múltiplas hipofisárias e de $30 \%$ a $40 \%$ nos com DGH isolada (30). Osório e cols. avaliaram 76 pacientes com DGH e correlacionaram achados da RM com níveis hormonais e mutações dos genes $G H-1$, receptor GHRH e PROP-1. Todos os pacientes com mutações tinham haste íntegra, enquanto a interrupção da haste estava presente em $74 \%$ dos pacientes sem mutação e neuro-hipófise ectópica em $87 \%$ destes. Nos pacientes com deficiências múltiplas, a avaliação hormonal era sugestiva de deficiência hipofisária nos casos de mutação do PROP 1 e PIT 1 e de deficiência hipotalâmica nos casos de interrupção de haste (31). Coutant e cols. analisaram a estatura final de 63 crianças com DGH idiopática tratadas e compararam com crianças com BEI (32 tratadas com GH e 51 
não-tratadas). Observaram que o único grupo que atingiu estatura final maior foi o dos deficientes com RM alterada. Os deficientes com RM normal atingiram estatura final semelhante aos pacientes com BEI (32). O hipersinal da neuro-hipófise pode estar ausente em 10\% dos indivíduos normais e em um estudo recente foi demonstrado que $20 \%$ dos pacientes com neuro-hipófise ectópica, $32 \%$ dos com hipoplasia hipofisária e $48 \%$ dos com RM normal apresentaram pico de GH superior a $10 \mathrm{mcg} / \mathrm{L}$ nos testes de estímulo no momento do reteste. O IGF-1 destes pacientes também era normal, apesar de significativamente mais baixo no grupo com neuro-hipófise ectópica do que nos sem alteração anatômica, e mais baixo nestes do que em um grupo controle (33).

\section{CONCLUSÕES}

Usualmente considera-se anormal o valor de teste diagnóstico quando este se encontra ao menos 2 DP distante da média observada em pessoas normais. O presente estudo, assim como a revisão da literatura, demonstraram que este critério não é útil para a triagem da DGH empregando a medida de IGF-1 (11). Considerando o IGF-1 (11) como exame de triagem (screening) que deve priorizar a sensibilidade, recomenda-se a utilização de redução de -1 DP comparado com a média para a idade do paciente como indicativa de DGH. Nesta situação, o aspecto de maior relevância volta a ser o quadro clínico e auxológico da criança, quando decidiu-se para qual paciente seria solicitada a medida do IGF-1. Neste caso, além da estatura atual, será muito importante considerar a VC e a presença de outros achados clínicos sugestivos de DGH.

Quanto ao pico de resposta do GH ao teste de estímulo, observamos que o ponto de corte de $10 \mathrm{mcg} / \mathrm{L}$, amplamente recomendado na literatura, produz número extremamente elevado de testes falsos-positivos $(46,1 \%)$ nos testes de GH clonidina sem priming, e especificidade relativamente baixa, entre $54,3 \%$ a $77,1 \%(11)$.

Em conclusão e considerando todos os aspectos discutidos, sugere-se abordagem prática para o diagnóstico da DGH em pacientes com BE: 1) inicialmente avaliar clínica e laboratorialmente a criança com BE para excluir doenças sistêmicas e/ou genéticas; 2) dosar o IGF-1 basal nas crianças com estatura abaixo do terceiro percentil ou quando houver diminuição da VC abaixo do percentil 25 ; 3) caso a criança não apresente outra causa para redução do IGF-1 (doença crônica, desnutrição) e apresentar valor de IGF-1 abaixo de -1 DP para a idade, submetê-la ao teste de estímulo da secreção do GH (clonidina/glucagon/insulina); 4) crianças que em dois testes de estímulo do $\mathrm{GH}$ não tenham resposta superior a 5 $\mathrm{mcg} / \mathrm{L}$, quando utilizado ensaio quimioluminescente mono-policlonal para medida do $\mathrm{GH}$, devem ser diagnosticadas como deficientes e tratadas; 5 ) crianças com IGF-1 abaixo de -1 DP e um teste de estímulo não responsivo na presença de fatores de risco, como irradiação prévia de SNC, quimioterapia, tumor ou outra doença hipotálamo-hipofisária, alteração de exame de imagem da região hipotálamo-hipofisária ou deficiência de outros hormônios hipofisários, também devem ser diagnosticadas como deficientes de $\mathrm{GH}$; 6) crianças com IGF-1 maior do que -1 DP dificilmente serão deficientes, porém devem ser acompanhadas para avaliar sua VC, que, se muito baixa, pode indicar a necessidade de reavaliação do eixo GH/IGF-1.

Finalizando, é importante considerar que não existe um único teste diagnóstico da DGH. Pelo contrário, o diagnóstico deve ser estabelecido pela análise de um conjunto de fatores: $\mathrm{BE}$, exclusão de doenças crônicas e síndromes genéticas, VC abaixo do percentil 25 , atraso de idade óssea, estatura abaixo do canal de crescimento relacionado à altura-alvo, presença de fatores de risco (irradiação prévia de SNC, quimioterapia, tumor ou outra doença hipotálamo-hipofisária), integridade dos demais eixos hipofisários, alteração de exame de imagem da região hipotálamo-hipofisária, valor do IGF-1 abaixo de -1 DP e valor do pico de resposta do $\mathrm{GH}$ a pelo menos dois estímulos abaixo de $5 \mathrm{mcg} / \mathrm{L}$. Assim, é elevado o valor preditivo positivo da agregação destas diversas alterações, em um mesmo paciente, de maneira que, nesta circunstância, podemos, inclusive, dispensar a necessidade de múltiplos testes de estímulo de GH para o estabelecimento do diagnóstico de sua deficiência.

\section{REFERÊNCIAS}

1. Roith D L, Bondy C, Yakar S, Liu J L, and Butler A. The somatomedin hypothesis: 2001. Endocr Rev. 2001;22(1):53-74.

2. Bussières L, Souberbielle JC, Pinto G, Adan L, Noel M, Brauner $R$. The use of insulin-like growth factor 1 reference values for the diagnosis of growth hormone deficiency in prepubertal children. Clin Endocrinol. (Oxf) 2000;52:735-9.

3. Juul A, Bang P, Hertel NT, Main K, Dalgaard P, Jorgensen K, et al. Serum insulin-like growth factor-i in 1030 healthy children, adolescents, and adults: relation to age, sex, stage of puberty, testicular size, and body mass index. J Clin Endocrinol Metab 1994;78(3):744-52. 
4. Juul A, Holm K, Kastrup KW, Pedersen SA, Michaelsen KM, Scheike $T$, et al. Free insulin-like growth factor I serum levels in 1430 healthy children and adults, and its diagnostic value in patients suspected of growth hormone deficiency. J Clin Endocrinol Metab. 1997;82:2497-502.

5. Reiter EO, Rosenfeld RG. Normal and aberrant growth. In: Kronenberg HM, Melmed S, Polonsky KS, Larsen PR, editors. Williams textbook of endocrinology. 11th ed. Saunders Elsevier; 2008. p 849-968.

6. Juul A, Moller S, Mosfeldt-Laursen E, Rasmussen MH, Scheike T, Pedersen SA, et al. The acid-labile subunit of human ternary insulin-like growth factor binding protein complex in serum: hepatosplanchnic release, diurnal variation, circulating concentrations in healthy subjects, and diagnostic use in patients with growth hormone deficiency. J Clin Endocrinol Metab. 1998;83:4408-15.

7. Growth Hormone Research Society. Consensus guidelines for the diagnosis and treatment of growth hormone (GH) deficiency in childhood and adolescence: summary statement of the GH Research Society. J Clin Endocrinol Metab. 2000;85(11):3990-3.

8. Saenger P. Editorials. Growth hormone in growth hormone deficiency. Start treatment early and give it for long enough. Br Med J. 2002;325:58-9.

9. Darzy KH, Pezzoli SS, Thorner MO, Shalet SM. Cranial irradiation and growth hormone neurosecretory dysfunction: a critical appraisal. J Clin Endocrinol Metab. 2007;92:1666-72.

10. Darzy KH, Aimaretti G, Wieringa G, Rao Gattamaneni H, Ghigo $\mathrm{E}$, Shalet SM. The usefulness of the combined growth hormone (GH)-releasing hormone and arginine stimulation test in the diagnosis of radiation-induced GH deficiency is dependent on the post-irradiation time interval. J Clin Endocrinol Metab. 2003;88:95-102.

11. De Paula LP. Estudos Prospectivos no diagnóstico diferencial da baixa estatura e da deficiência de hormônio de crescimento em crianças. Orientador: Mauro A Czepielewski. [Tese: doutorado] Faculdade de Medicina, UFRGS, 2007. p. 103.

12. Nunez SB, Municchi G, Barnes KM, Rose SR. Insulin-like growth factor I (IGF-1) and IGF-binding protein-3 concentrations compared to stimulated and night growth hormone in the evaluation of short children - a clinical research center study. J Clin Endocrinol Metab. 1996;81:1927-32.

13. Juul A, Kastrup KW, Pedersen SA, Skakkebaek NE. Growth hormone $(\mathrm{GH})$ provocative retesting of 108 young adults with childhood-onset GH deficiency and the diagnostic value of insulin-like growth factor I (IGF-1) and IGF-binding protein-3. J Clin Endocrinol Metab. 1997;82:1195-201.

14. Kawai N, Kanzaki S, Takano-Watou S, Tada C, Yamanaka Y, Miyata T, et al. Serum free insulin-like growth factor I (IGF-I), total IGF-I, and IGF-binding protein-3 concentrations in normal children and children with growth hormone deficiency. J Clin Endocrinol Metab. 1999;84(1):82-9.

15. Rosenfeld RG, Wilson DM, Lee PD, Hintz RL. Insulin-like growth factors I and II in evaluation of growth retardation. J Pediatr 1986;109:428-33.

16. Ranke MB, Schweizer R, Elmlinger MW, Weber K, Binder G, Schwarze CP, Wollmann HA. Significance of basal IGF-1, IGFBP-3 and IGFBP-2 measurements in the diagnostics of short stature in children. Horm Res. 2000;54:60-68.

17. Mitchell H, Dattani MT, Nanduri V, Hinmarsh PC, Preece MA, Brook CG. Failure of IGF-1 and IGFBP-3 to diagnose growth hormone insufficiency. Arch Dis Child. 1999;80:443-7.
18. Tassoni P, Cacciari E, Cau M, Colli C, Tosi M, Zucchini S, et al. Variability of growth hormone response to pharmacological and sleep tests performed twice in short children. J Clin Endocrinol Metab. 1990;71:230-4.

19. Zadik Z, Chalew SA, Gilula Z, Kowarski A. Reproducibility of growth hormone testing procedures: A comparison between 24-hour integrated concentration and pharmacological stimuIation. J Clin Endocrinol Metab. 1990;71:1127-30.

20. Marin G, Domené HM, Barnes KM, Blackwell BJ, Cassorla FG, Cutler Jr GB. The effects of estrogen priming and puberty on the growth hormone response to standardized treadmill exercise and arginine-insulin in normal girls and boys. J Clin Endocrinol Metab. 1994;79(2):537-41.

21. Rosenfeld RG, Albertsson-Wikland K, Cassorla F, Frasier SD, Hasegawa $Y$, Hintz RL, et al. Diagnostic controversy: the diagnosis of childhood growth hormone deficiency revisited. $J$ Clin Endocrinol Metab. 1995;80(5):1532-40.

22. Chanoine JP, Rebuffat E, Kahn A, Bergmann P, Van Vliet G. Glucose, growth hormone, cortisol, and insulin responses to glucagon injection in normal infants, aged 0.5- 12 months. J Clin Endocrinol Metab. 1995;80:30-2.

23. Ghigo E, Bellone J, Aimaretti S, Bellone S, Loche S, Cappa M, et al. Reliability of provocative tests to assess growth hormone secretory status. Study in 472 normally growing children. $J$ Clin Endocrinol Metab. 1996;81:3323-7.

24. Celniker AC, Chen AB, Wert Jr RM, Sherman BM. Variability in the quantitation of circulating growth hormone using commercial immunoassays. J Clin Endocrinol Metab. 1989;68(2):469-76.

25. Tauber M. Growth hormone testing in KIGS. In: Ranke MB, Price DA, Reiter EO, editors. Growth hormone therapy in pediatrics - 20 years of KIGS. Basel, Karger, 2007: 54-85.

26. Cacciari E, Tassoni P, Cicognani A, Pirazzoli P, Salardi S, Balsamo A, et al. Value and limits of pharmacological and physiological tests to diagnose growth hormone (GH) deficiency and predict therapy response: first and second retesting during replacement therapy of patients defined as GH deficient. J Clin Endocrinol Metab. 1994;79(6):1663-9.

27. Silva EGP, SIhessarenko N, Arnhold IJP, Batista MC, Estefan V, Osório MGF, et al. GH values after clonidine stimulation measured by immunofluorometric assay in normal prepubertal children and GH-deficient patients. Horm Res. 2003;59:229-33.

28. Martínez AS, Domené HM, Ropelato G, Jasper HG, Pennisi Pa, Escobar ME, Heinrich JJ. Estrogen priming effect on growth hormone $(\mathrm{GH})$ provocative test: A useful tool for the diagnosis of GH deficiency. J Clin Endocrinol Metab 2000; 85: 4168-72.

29. Chaler EA, Rivarola MA, Guerci B, Ciaccio M, Costanzo M, Travaglino $P$, et al. Differences in serum $G H$ cut-off values for pharmacological tests of $\mathrm{GH}$ secretion depend on the serum $\mathrm{GH}$ method. Clinical validation from the growth velocity score during the first year of treatment. Horm Res. 2006; 6:231-5.

30. Maghnie M, di Lorgi N, Rossi A, Gastaldi R, Tortori-Donati $P_{\text {, }}$ Lorini R. Neuroimaging in growth hormone deficiency. In: Ranke MB, Price DA, Reiter EO (eds): Growth hormone therapy in pediatrics -20 years of KIGS. Basel, Karger, 2007: 93-107.

31. Osório MGF, Marui S, Jorge AAL, Latronico AC, Lo LSS, Leite $\mathrm{CC}$, et al. Pituitary magnetic resonance imaging and function in patients with growth hormone deficiency with and without mutations in GHRH-R, GH-1, or PROP-1 genes. J Clin Endocrinol Metab. 2002;87:5076-84.

32. Coutant R, Rouleau S, Despert F, Magontier N, Loisel D, Limal JM. Growth and adult height in GH-treated children with no- 
nacquired GH deficiency and idiopathic short stature: The influence of pituitary magnetic resonance imaging findings. $J$ Clin Endocrinol Metab. 2001;86:4649-54.

33. Gelwane G, Garel C, Chevenne D, Armoogum P, Simon D, Czernichow $P$, Léger J. Subnormal serum insulin-like growth factor-1 levels in young adults with childhood-onset nonacquired growth hormone (GH) deficiency who recover normal GH secretion may indicate less severe but persistent pituitary failure. J Clin Endocrinol Metab. 2007;92:3788-95.
Endereço para correspondência:

Leila Pedroso de Paula

Av. Lucas de Oliveira, 1937, apto. 502

90035-003 Porto Alegre, RS

E-mail: endo.endoped@yahoo.com.br 